\title{
Doppler effect in rotating systems and Mössbauer rotor experiments
}

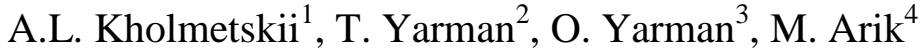 \\ ${ }^{1}$ Department of Physics, Belarusian State University, 4 Nezavisimosti Avenue, 220030 Minsk, \\ Belarus \\ ${ }^{2}$ Department of Engineering, Istanbul Okan University, Akfirat, Istanbul \\ ${ }^{3}$ Istanbul University, Istanbul, Turkey \\ ${ }^{4}$ Bogazici University, Istanbul, Turkey
}

\begin{abstract}
We address once more the problem of the physical interpretation of the Mössbauer effect in a rotating system in the view of the recently discovered extra energy shift (EES) between emitted and absorbed resonant radiation, which emerges in addition to the usual second order Doppler shift for an orbiting absorber. We highlight that both fractional components of the total energy shift have the same sign and correspond to the blueshift of the resonant radiation propagating from the rotational axis to the rotor rim. We indicate a possible way of understanding the origin of the EES in terms of combining metric effects in rotating systems with the quantum mechanical description of resonant nuclei confined in crystal cells by taking into account the recoil-free essence of the Mössbauer effect, where no energy is transmitted to the source (absorber) during the emission (absorption) of resonant radiation.
\end{abstract}

\section{Introduction}

In the past decade, Mössbauer experiments in a rotating system attracted considerable attention due to our disclosure of an extra energy shift (following the usual transverse Doppler shift) between the resonant lines of the emitter and the absorber under different radial coordinates [1-4]. The presence of such an extra energy shift (hereinafter abbreviated as the EES) was first revealed in the re-analysis [1] of Kündig's experiment [5], and further confirmed by modern measurements [2-4].

In particular for a configuration, where the source of resonant radiation is fixed on the rotor axis while the resonant absorber is located on the rotor rim, instead of the standard relativistic prediction (the second order Doppler shift)

$$
\frac{v_{s}-v_{a}}{v_{s}}=\frac{\Delta v}{v_{s}}=-\frac{1}{2} \frac{u^{2}}{c^{2}}
$$

we obtained

$$
\frac{\Delta v}{v_{s}}=-k \frac{u^{2}}{c^{2}}
$$

with the coefficient $k$ being substantially larger than 0.5 , as originally predicted by T. Yarman [6]. Here $v_{s}\left(v_{a}\right)$ is the frequency of the resonant radiation for the source (absorber), $u$ is the tangential velocity of the absorber, and $c$ is the light velocity in vacuum.

We remind that our re-estimation [1] of the experiment by Kündig [5] yields the inequality $k>0.6$, while our experiments [2-4] both give the value of $k=2 / 3$ with a measurement precision of about $5 \%$.

These results unambiguously corroborate the presence of the EES between emitted and absorbed resonant radiation in a rotating system, and the clarification of its physical meaning becomes a topical problem. 
At the moment, there are several attempts to explain the origin of the EES, and most of them remain unsuccessful (see, e.g., [7]). Among such attempts, we would like to mention the hypothesis about the existence of a limited acceleration in nature (see, e.g., [8]) and some exotic extensions of relativity theory (e.g., [9]), whereby their failure once again accentuates the need to look for a consistent explanation of the EES under the formalism of general relativity (GR) as applied to rotating systems.

At the same time, all available attempts to explain the essence of the EES in GR contain errors (for a brief review, see [7]), so that the problem remains open to date.

A recent endeavor to understand the origin of the EES through the formalism of GR was attempted in the paper by Podosenov et al. [10]; however, as we pointed out in [11], the experimental scheme assumed in [10] has never been realized in any of the known Mössbauer rotor experiments.

Nevertheless, said authors went on to publish the erratum [12], where they eliminated some technical errors in [10], but still avoided responding to our principal criticism [11].

In this respect, in section 2, we first focus on the correct interpretation of the second order Doppler effect in rotating systems with an adequate definition of such concepts as "characteristic energy" of a resonant source and an absorber, frequency shift of the resonant radiation in a rotating system, as well as the time dilation effect for the co-rotating resonant source and resonant absorber.

Then, in section 3, we discuss the results obtained and indicate a possible way to clarify the physical meaning of the EES.

\section{The Mössbauer effect in a rotating system: Analysis and interpretation}

In the analysis of Mössbauer experiments in a rotating system, Podosenov et al. [10] applied the Langevin metric

$$
d s^{\prime 2}=\left(1-\frac{r^{2} \omega^{2}}{c^{2}}\right) c^{2} d t^{2}-2 \omega r^{2} d \phi^{\prime} d t^{\prime}-d r^{2}-r^{, 2} d \phi^{, 2}-d z^{, 2},
$$

which is obtained by transforming the cylindrical coordinates from a non-rotating inertial frame $\mathrm{K}(t, r, \phi, z)$ to a rotating frame $\mathrm{K}_{\mathrm{r}}\left(t^{\prime}, r^{\prime}, \phi^{\prime}, z^{\prime}\right)$

$$
t=t^{\prime}, r=r^{\prime}, \phi=\phi^{\prime}+\omega t^{\prime}, z=z^{\prime} \text {. }
$$

Then, they considered the propagation of the resonant $\gamma$-quanta from a source on the rotor axis (where $r=0$ ) to an absorber at the rotor edge (where $r=R$ ) and assumed that such $\gamma$-quanta propagate along a straight line for a rotating observer, insofar as implying the equality

$\phi^{\prime}=0$.

Further, by combining eqs. (3) and (5), Podosenov et al. obtained their own expression

$$
v_{a}=v_{s}\left(1-\frac{2}{3} \frac{u^{2}}{c^{2}}\right)
$$

(in the notations of the present paper) for the frequency of the resonant radiation passing through a resonant absorber [10]. Hence, Podosenov et al. arrived at the relative frequency shift expression

$$
\frac{v_{s}-v_{a}}{v_{s}}=\frac{\Delta v}{v_{s}}=\frac{2}{3} \frac{u^{2}}{c^{2}}
$$

which, however, differs from eq. (2) due to its sign.

Thus, eq. (7) corresponds to the redshift of radiation in contrast to the blueshift described by eq. (2).

In our paper [11], we have already clarified the origin of the disagreement between eqs. (2) and (7), emphasizing that, in all of the Mössbauer rotor experiments performed up to date - including the experiment by Kündig [5] and our own experiments [2-4], the resonant $\gamma$-quanta emitted by the source propagate in the vacuum chamber of the rotor along a straight line as seen 
by a laboratory observer, while the constraint (5) adopted by Podosenov et al. in [10] corresponds to the propagation of resonant $\gamma$-quanta from the source to the absorber along a straight line for a co-rotating observer.

This means that, for a laboratory observer, under the constraint (5), the resonant radiation would propagate in empty space along a curved trajectory!

This is obviously nonsensical, unless a special very thin guide of resonant $\gamma$-quanta is utilized to connect the co-rotating source and absorber.

However, such guides for $\gamma$-radiation were not (and could not be) applied to any of the known Mössbauer rotor experiments because they are not even invented or thought of yet.

Hence, the expression (7) derived by Podosenov et al. in [10] has no relation to any of the performed Mössbauer rotor experiments [11].

Still, in the recent erratum [12], Podosenov et al. continue to keep silence regarding our criticism of their impractical constraint (5). Moreover, concerning the disagreement between eqs. (2) and (7), they write "Note that we obtain redshift in accordance with Kündig experiment [3] and with canonical GTR prediction [4-6]). (Here references are numbered according to [10]).

This claim also deserves a separate consideration.

First, we address the paper by Kündig [5] and find his equation (1) for the fractional energy shift of the resonant radiation in the inertial frame of the source:

$$
\left(E_{A}-E_{S}\right) / E_{S}=-R_{A}{ }^{2} \omega^{2} / 2 c^{2} .
$$

Here, Kündig defined $E_{A}$ and $E_{S}$ as "...the characteristic energies of the absorber and the source", while the product $R_{A} \omega$ stands for the tangential velocity of the absorber, designated in our equations (1) and (2) as $u$.

Thus, eq. (8) indicates that $E_{A}<E_{S}$, which means that for a laboratory observer, the maximum of the resonant line of the orbiting absorber is shifted towards lower energies. Therefore, in a rotating frame, attached to the absorber - where $E_{A}$ is a fixed proper value $(e . g$., it coincides with the maximum of the resonant line) - the frequency of the radiation from the source is conversely shifted towards higher energies. This means that the co-rotating observer registers a blueshift of resonant radiation, which is in obvious contradiction with eq. (7) by Podosenov et al., that assumes a redshift.

Unfortunately, in the subsequent equation (3) of [5]

$$
v_{A}=v_{S}\left(1+2 \Phi / c^{2}\right)^{1 / 2} \approx v_{S}\left(1+\Phi / c^{2}\right),
$$

written in the rotating frame (where $\Phi=-R_{A}{ }^{2} \omega^{2} / 2 c^{2}$ is the effective gravitational potential), Kündig made a mistake, coming at odds with his own correct equation (8). Indeed, as we found above, eq. (8) leads to the inequality $v_{A}>v_{S}$, corresponding to the blueshift of the resonant radiation, whereas eq. (9) yields the reverse inequality $v_{A}<v_{S}$ (the redshift).

In order to show the flawed character of eq. (9), we address the Langevin metric (3) and determine the relationship between the time intervals $d t_{S}^{\prime}$ (at $r^{\prime}=0, d r^{\prime}=d z^{\prime}=d \phi^{\prime}=0$ ) and $d t_{A}^{\prime}$ (at $\left.r^{\prime}=R, d r^{\prime}=d z^{\prime}=d \phi^{\prime}=0\right)$ of the rotating system with a sufficient accuracy $c^{-2}$ :

$$
d t_{A}^{\prime}=d t_{S}^{\prime}\left(1-R_{A}{ }^{2} \omega^{2} / c^{2}\right)^{1 / 2} \approx d t_{S}^{\prime}\left(1-R_{A}{ }^{2} \omega^{2} / c^{2}\right) \text {. }
$$

Since the relationship between the corresponding frequencies is reversible to (10), we derive in the designations by Kündig:

$$
v_{A}=v_{S}\left(1+2 \Phi / c^{2}\right)^{-1 / 2} \approx v_{S}\left(1-\Phi / c^{2}\right)=v_{S}\left(1+R_{A}^{2} \omega^{2} / c^{2}\right)
$$

Thus, replacing the faulty equation (9) with the correct equation (11), we get the inequality $v_{A}>v_{S}$, indicating indeed a blueshift of the resonant radiation for the configuration where the resonant source is fixed on the rotor axis, and the resonant absorber is orbiting at the rotor rim.

At the same time, the mistake committed by Kündig in equation (9) (which is eq. (3) of [5]) does not affect anyway the validity of his measurement results that definitely indicate the blueshift of resonant radiation as referred to the co-rotating observer. 
In order to demonstrate that this is actually the case, we reproduce in Fig. 1 the Figure 3 from [5], which shows the measured resonant absorption curves at different rotational speeds of the rotor.

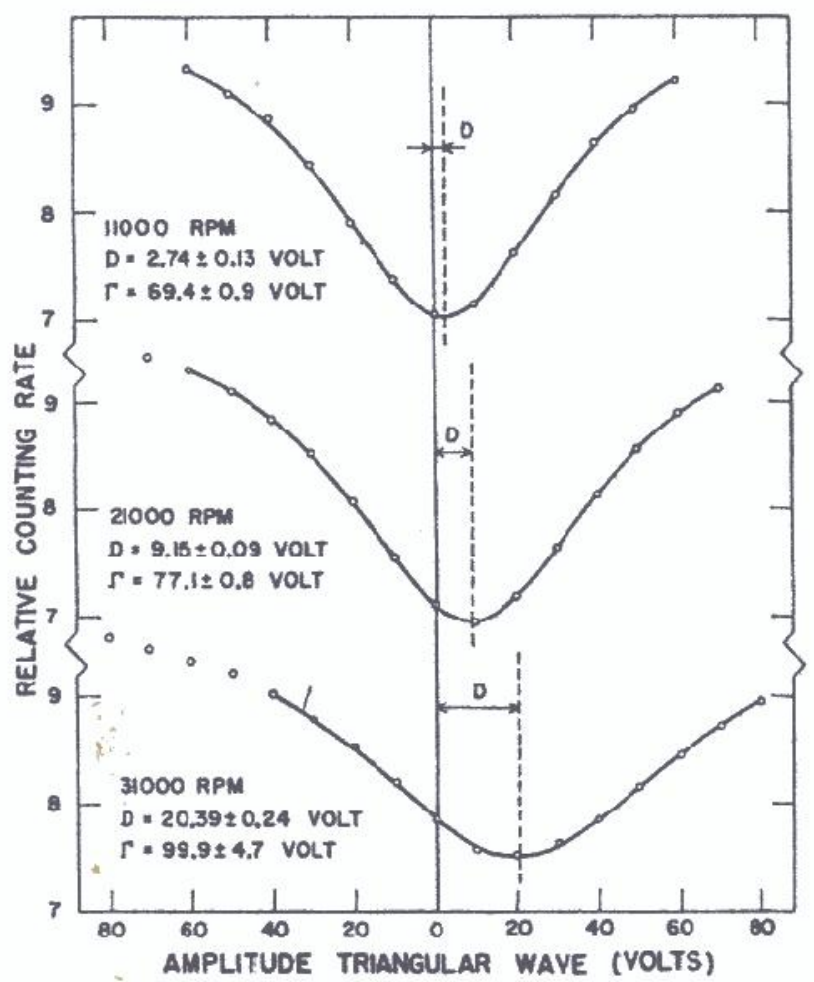

FIG, 3. Typical resonance curves. The amplitude of the triangular wave is proportional to the linear velocity of the source with respect to the absorber. The left (right) side of the plot corresponds to a motion of the source toward (away from) the absorber. The plotted curves are the fitted Lorentz curves normalized to 10 at $\vartheta=\infty . \Gamma$ is the full width of the resonance line. With increasing speed of the rotor a considerable broadening of the resonance line was observed. The statistical errors of the points are smaller than the circles.

Fig. 1. Scanned copy of Fig. 3 from the paper by Kündig [5].

Recall that the resonant curves in Kündig's experiment were measured with the reciprocating motion of the resonant source on the rotational axis towards and backwards with respect to the orbiting resonant absorber fixed at the rotor edge.

In this configuration, the measured shape and position of resonant absorption lines in Fig. 1 is defined by a mixture of two effects: The first effect is frame-independent, and represents a linear Doppler shift of the emitted resonant radiation due to the oscillations of the source towards and backwards from the absorber; the manifestation of the second effect is frame-dependent, and, in the laboratory frame, it corresponds to the second order Doppler shift for the orbiting resonant absorber, while in the rotating frame it can be interpreted as a frequency shift of the resonant radiation due to the variation of the effective gravitational potential.

Under these circumstances, we have to understand what kind of frequency shift - redshift or blueshift - corresponds to the experimental data of Kündig in Fig. 1 under their consideration of both reference frames introduced above. 
First of all, we point out that, according to Fig. 1, the maximum of the resonant line is shifted to the right with increasing rotational speed. In the caption to this figure, Kündig indicates that the right side of the plot corresponds to the motion of the source away from the absorber when the sign of the linear Doppler effect is negative. Therefore, in a rotating frame, the counterbalancing frequency shift of the resonant radiation due to the effective gravitational potential is positive and thus represents a blueshift.

In such a way, we invalidate the claim by Podosenov et al. [10] presented above: "Note that we obtain redshift in accordance with Kündig experiment...". As we have explained with the data in Fig. 1, Kündig measured instead the blueshift of the resonant radiation, as referred to the orbiting absorber.

We notice that the blueshift of the resonant radiation in Mössbauer rotor experiments with a source on the rotational axis can also be obtained for a laboratory observer, where it is interpreted through the second order Doppler effect. Indeed, when the source and absorber are both fixed on the rotor, no additional contribution to the linear Doppler effect due to their rotation arises as seen by a laboratory observer (see, e.g. [7]); therefore, the relative shift of the resonant lines of the source and the absorber is determined only by the second order Doppler effect emerging due to the time dilation for the orbiting absorber.

Indeed, suppose that a source on the rotating axis (origin of coordinates) emits electromagnetic radiation with $N$ wavelengths per each unit time interval $t_{s}$, which corresponds to the frequency $v_{s}=N / t_{s}$. When this radiation reaches the resonant absorber, the attached observer counts the same number of wavelengths $N$, but for a shorter time interval $t_{a}=t_{s} / \gamma$ (where $\gamma$ is the Lorentz factor) due to the relativistic dilation of its time, as seen by a laboratory observer. Consequently, the frequency of the resonant radiation measured at the absorber is equal to

$v_{a}=N / t_{a}=\gamma N / t_{s}=\gamma \nu_{s}$,

which again signifies a blueshift of the resonant radiation $\left(\mathrm{v}_{a}>\mathrm{v}_{s}\right)$.

Finally, we consider the remaining part of the erroneous claim by Podosenov et al. presented above: "Note that we obtain redshift in accordance with...canonical GTR prediction [46]".

In order to demonstrate the fallacy of this phrase, it is enough to look at the readily accessible ref. [6] of [10] (which is ref. [13] of our paper), where we find the following relationship between the frequency of emitted $\left(\omega_{s}\right)$ and absorbed $\left(\omega_{a}\right)$ radiation (eq. (11) of [13]):

$$
\frac{\omega_{a}}{\omega_{s}}=\frac{\sqrt{1-\Omega^{2} R_{s}^{2} / c^{2}}}{\sqrt{1-\Omega^{2} R_{a}^{2} / c^{2}}},
$$

where $\Omega$ is the angular velocity of the rotor, and $R_{a}\left(R_{s}\right)$ is the radial coordinate of the absorber (source) of $\gamma$-radiation.

One can see that at $R_{a}>R_{s}$ (which is the case for experiments [2-5]), one gets $\omega_{a}>\omega_{s}$, which corresponds to the blueshift of the resonant radiation, in contrast to the claim in [10]. We further notice that at $R_{s}=0$ (when the source is fixed on the rotational axis), and with the designation $\Omega R_{a}=u$, we arrive at eq. (1) to an accuracy of $c^{-2}$.

\section{Discussion}

We have shown once again that the approach by Podosenov et al. [10, 12] aimed at explaining the origin of the EES on the basis of their constraint (5) has no relation with the actual implementation of all Mössbauer rotor experiments, where resonant radiation propagated in empty space from a source on the rotating axis to an absorber at the rotor rim along the straight line when

$$
\phi=0
$$

for the laboratory observer. 
As we have shown in [11], the replacement of the constraint (5) by the equality (13), with its further substitution into eqs. (4c) and (3) gives

$$
d s^{\prime 2}=c^{2} d t^{\prime 2}-d r^{\prime 2}-d z^{\prime 2}
$$

which, at $d s^{\prime}=0$ (propagation of resonant $\gamma$-quanta in empty space), yields the trivial equality

$$
\frac{d r^{\prime}}{d t^{\prime}}=\frac{d r}{d t}=c \text {. }
$$

In fact, eq. (15) signifies the failure of standard relativistic approaches to explain the origin of EES in Mössbauer rotor experiments.

In this respect, it seems promising to re-visit our idea already outlined in [14], where we proposed to explicitly take into account the recoil-free origin of the Mössbauer effect along with the relativistic effects in a rotating system. The recoil-free interaction of resonant nuclei with crystals of the source and the absorber means that emission (absorption) events do not affect the total energy of the source and the absorber, and therefore, they can be considered as a unified quantum mechanical system. In this case, the possible dependence of the energy levels of the resonant nuclei confined in crystals cells on the effective gravitational potential could contribute to the observed energy shift between emission and absorption lines and hence explain the origin of the EES.

If this idea is principally correct, then, in the purely classical case of rotation, no EES should arise, which seems to be actually the case [15].

However, a detailed analysis of this problem lies beyond the scope of the present contribution.

\section{Declaration of competing interest}

The authors declare that they have no known competing financial interests or personal relation-ships that could have appeared to influence the work reported in this paper.

\section{References}

[1] A.L. Kholmetskii, T. Yarman, O.V. Missevitch, Phys. Scr. 78 (2008) 035302.

[2] A.L. Kholmetskii, T. Yarman, O.V. Missevitch, B.I. Rogozev, Phys. Scr. 79 (2009) 065007.

[3] A.L. Kholmetskii, T. Yarman, M. Arik and O.V. Missevitch, AIP Conf. Proc. 1648 (2015) 510011.

[4] T. Yarman, A.L. Kholmetskii, M. Arik, B. Akkus, Y. Öktem, L.A. Susam, O.V. Missevitch, Can. J. Phys. 94 (2016) 780.

[5] W. Kündig. Phys. Rev. 129 (1963) 2371.

[6] T. Yarman, Ann. Found. De Broglie 29 (2004) 459.

[7] A.L. Kholmetskii, T. Yarman, O. Yarman, M. Arik, J. Synchr. Rad. 28 (2021) 78.

[8] E.R. Caianiello, Lett Nuov. Cim. 32 (1981) 65.

[9] E. Benedetto, A. Feoli, Eur. Phys. J. Plus 133 (2018) 53.

[10] S.A. Podosenov, J. Foukzon, E.R. Men'kova, Ann. Phys. 413 (2020) 168047.

[11] A.L. Kholmetskii, T. Yarman, O. Yarman, M. Arik, Ann. Phys. 418 (2020) 168191.

[12] S.A. Podosenov, J. Foukzon, E.R. Men'kova, Ann. Phys. 423 (2020) 168329.

[13] G. Giuliani, Eur. J. Phys. 35 (2014) 025015.

[14] T. Yarman, A.L. Kholmetskii, M. Arik, Eur. Phys. J. Plus 130 (2015) 191.

[15] T. Yarman, A.L. Kholmetskii, O. Yarman, M. Arik, Ann. Phys. 423 (2020) 168337. 